\title{
A Spatial Contextual Postclassification Method for Preserving Linear Objects in Multispectral Imagery
}

\author{
Borja Rodríguez-Cuenca, Student Member, IEEE, Jose A. Malpica, and Maria C. Alonso
}

\begin{abstract}
Classification of remote sensing multispectral data is important for segmenting images and thematic mapping and is generally the first step in feature extraction. Per-pixel classification, based on spectral information alone, generally produces noisy classification results. The introduction of spatial information has been shown to be beneficial in removing most of this noise. Probabilistic label relaxation (PLR) has proved to be advantageous using second-order statistics; here, we present a modified contextual probabilistic relaxation method based on imposing directional information in the joint probability with third-order statistics. The proposed method was tested in synthetic images and real images; the results are compared with a "Majority" algorithm and the classical PLR method. The proposed third-order method gives the best results, both visually and numerically.
\end{abstract}

Index Terms-Classification smoothing, contextual classification, relaxation methods, remote sensing.

\section{INTRODUCTION}

$\mathbf{C}$ LASSIFICATION of multispectral image data based on spectral information is used in analyzing remotely sensed data. The objective of the classification process is to categorize all pixels in a satellite or aerial image into one of several land cover classes. This categorized data may then be used to produce thematic maps of the existing land cover present in an image. There are two main classification methods: supervised and unsupervised. In the former, samples of the information classes (land cover type) of interest in the image, called training sites, are identified [1]. From these training areas, statistics are first calculated and then used to classify each independent pixel of the entire image being examined. Decision rules are then applied; these can be nonparametric, such as minimum Euclidean distance, or parametric, such as Gaussian maximum likelihood (ML). In unsupervised classification, often referred to simply as cluster analysis, a computer algorithm partitions the image into self-defining spectral clusters. Supervised or unsupervised classification methods have generally used only information obtained from individual pixels; therefore, the final thematic maps tend to be somewhat noisy, in what is known as saltand-pepper classified images [2], [3]. Pixels generally belong

Manuscript received April 14, 2011; revised November 16, 2011; accepted April 1, 2012. Date of publication June 1, 2012; date of current version December 19, 2012. This work was supported in part by the Spanish Ministerio de Ciencia e Innovación under project CGL2010-15357 and in part by the University of Alcalá under project UAH 2011/EXP-031.

The authors are with the Department of Mathematics, University of Alcalá, 28871 Alcalá de Henares, Spain (e-mail: borja.rodriguezc@uah.es; josea. malpica@uah.es; mconcepcion.alonso@uah.es).

Color versions of one or more of the figures in this paper are available online at http://ieeexplore.iee.org.

Digital Object Identifier 10.1109/TGRS.2012.2197756 to cover types that form a geographic region or cartographic entity; consequently, pixels that are close together are more strongly related than those that are spatially distant. A normal approach in identifying land cover classes is to complement the use of spectral information with spatial information obtained from neighboring pixels.

Improving per-pixel classification by incorporating both spatial and spectral information involves a two-stage process: First, a spatial filter is applied to achieve more homogeneous regions; second, a per-pixel classification algorithm is applied, as reported by Yildirim et al. [4]. These authors applied an ML algorithm to classify land cover and achieved an improvement over the extraction and classification of homogeneous object algorithm presented by Ketting and Landgrebe [5].

It is more common to apply the opposite procedurepostprocessing-rather than preprocessing, i.e., classifying by first using a per-pixel classification algorithm and then performing a postclassification operation. One of the simplest postprocessing operations is the application of a Majority filter [6], [7]. To conduct this operation, a moving window is passed over each pixel in the classified image. If the class assigned to the central pixel in the window is not the majority class of the window, the pixel's class is changed to the majority class. If there is no majority class, the identity of the center pixel is not changed. As the window progresses through the image, the original class from the previous classified image is used, not the assigned class as modified from the previous window position [8]. Some authors have applied modifications to the Majority filter and achieved some improvement, such as [9], which used a Landsat Thematic Mapper image with an adaptive Majority filter, resulting in some reduction in classification errors.

In recent decades, several approaches have been adopted for incorporating contextual information into the classification of remote sensing fields, such as Markov random fields (MRFs) [10]-[14], knowledge-based methods or fuzzy methods [15][17], probabilistic label relaxation (PLR) [18], [19], and hybrids, combining other methods [20]. In this paper, the term contextual information will refer only to spatial information, even though this concept could also be extended to other types of miscellaneous information (ancillary data) associated with the current pixels, as proposed by some of the aforementioned studies.

In postprocessing methods, linear features are usually removed when trying to reduce the speckled appearance in a classified image, as found by Myeong et al. [21]. These authors observed this phenomenon when applying the Majority filter in postprocessing. In this paper, we develop a postprocessing approach that aims to preserve linear features.

This paper is organized as follows. Higher order statistics are described in Section II. The methodology applied with the 
proposed method is described in Section III. The results obtained using synthetic and real images are presented in Section IV. Finally, our conclusions are given in Section V.

\section{Higher Order Statistics With Probabilistic LABELING RELAXATION}

Natural images exhibit statistical regularities that differentiate them from images in which pixels have been generated randomly; moreover, the human visual system appears to have evolved to exploit such statistical regularities. Many studies support the notion that our visual system presents an efficient means for coding the statistical structures found in nature [22], [23]. First-order statistics deal with single pixels and do not take into account relationships between neighboring pixels in an image. This first order implicates the histogram, mean, standard deviation, skew, and kurtosis of gray levels in an image. Second-order statistics examine relationships and regularities between pairs of pixels in an image. Examples include image gradients and power spectra, which are computed in Fourier space and are related to autocorrelation functions. Third-order statistics examine ternaries of pixels, as in the work of Gagalowitz and Ma [24], using co-occurrence matrices; these authors showed that such a third-order model was able to capture most of the information about macroscopic planar textures such as wool, sand, etc.

Studies on PLR focus on measuring second-order statistics or the covariation between the properties of paired pixels, such as their grayscale levels [19]. We show that it is practical to directly measure higher order statistics using the strategy of estimating the probability along different directions. Structural information about linear features can be retrieved through statistics of triples of pixel values (third-order statistics).

In the following equations, the procedure for supervised classification is considered from a statistical perspective. Let the classes be

$$
\omega_{i}, \quad i=1, \ldots, C
$$

where $C$ is the total number of classes.

$$
p_{m}\left(\frac{\omega_{i}}{\aleph_{m}}\right), \quad i=1, \ldots, C
$$

is the probability of class $\omega_{i}$ occurring, given that pixel $m$ and the neighborhood $\aleph_{m}$ surrounding pixel $m$ have been observed. In principle, a label is influenced by the real label of all other labels in the image, but in order to model the phenomenon of spatial context, we will here suppose that a label is influenced only by a few close neighbor pixels. We also suppose that this property of being influenced by only a small neighborhood is independent of the position in the image of the actual pixels. This is similar to the properties established in MRF [25].

Pixel $m$ is assigned to class $\omega_{i}$ if

$$
p_{m}\left(\frac{\omega_{i}}{\aleph_{m}}\right) \geq p_{m}\left(\frac{\omega_{j}}{\aleph_{m}}\right) \quad \forall i \neq j
$$

From Bayes' theorem, we have

$$
p_{m}\left(\frac{\omega_{i}}{\aleph_{m}}\right) \propto p_{\omega_{i}}(m) \cdot p\left(\frac{\omega_{i}}{\aleph_{m}}\right)
$$

where $p_{\omega_{i}}(m)$ is the class conditional probability for class $\omega_{i}$ given pixel $m$ and $p\left(\omega_{i} / \aleph_{m}\right)$ is the prior probability of the $\omega_{i}$ class for neighborhood $\aleph_{m}$. This is a measure of how appropriate it is to assign pixel $m$ to class $\omega_{i}$, in view of the current neighborhood surrounding $m$, defined as $\aleph_{m}$.

Following Richards and Jia [26], the question is how to find a value for $p\left(\omega_{i} / \aleph_{m}\right)$. These authors have modeled this probability by both MRF and PLR with Dempster-Shafer; they found the latter to be better than the former. We will present a modification of the latter algorithm with a third-order statistic. Herein, the original PLR as applied by Richards and Jia [26] will be called PLR2 (as it utilizes second-order statistics), and our proposed method will be called PLR3.

\section{Mahalanobis Classifier and Labeling Relaxation PLR2}

The term $p\left(\omega_{i} / \aleph_{m}\right)$ in (3) is the initial estimate of the probability of each pixel's label for a neighborhood $\aleph_{m}$. These probabilities can be assigned from a previous classification based on pixel information alone. In our case, this was done using the Mahalanobis classifier. Per-pixel classification approaches, such as minimum Euclidean distance and ML, have been widely used in many remote sensing applications. The Mahalanobis classifier has been widely applied in the remote sensing community [19], [27]-[29]. It is a derivation of the ML discriminant function, specifically when the prior probabilities are considered to be equal [15]. The Mahalanobis distance is given by

$$
\left(x-m_{i}\right)^{t} \sum^{-1}\left(x-m_{i}\right)
$$

where $m_{i}$ is the mean for class $\omega_{i}$ and $\sum$ is the covariance matrix. Richards stated that "The maximum likelihood classifier can be regarded as a minimum distance measure that is direction sensitive and modified according to class." Simple minimum Euclidean distance classifiers have some limitations that can be overcome using a Mahalanobis metric. In particular, this can often address problems caused by poorly scaled or highly correlated features [30].

Label relaxation is an iterative heuristic technique that extracts contextual information from an image to reduce the ambiguity of predetermined labeling. Relaxation labeling utilizes two sources of information: an initial labeling for $p\left(\omega_{i} / \aleph_{m}\right)$ and information embedded in spatial context. Several of the early PLR algorithms were proposed in the late 1970s and 1980s. Among the most popular are probabilistic labeling relaxation [31] and the supervised extraction and classification of homogeneous objects developed by Landgrebe [32] and Richards et al. [33].

Following Richards [19]

$$
p_{m}^{k}\left(\frac{\omega_{i}}{\aleph_{m}}\right)
$$

is an estimate of the probability that, on the $k$ th iteration, the label or class of the pixel $m$ is $\omega_{i}$. An iterative process was constructed in order to progressively modify the initial probability assigned to pixel $m$

$$
p_{m}^{k+1}\left(\frac{\omega_{i}}{\aleph_{m}}\right)=\frac{p_{m}^{k}\left(\omega_{i}\right) \cdot Q_{m}^{k}\left(\omega_{i}\right)}{\sum_{j} p_{m}^{k}\left(\omega_{j}\right) \cdot Q_{m}^{k}\left(\omega_{j}\right)}
$$



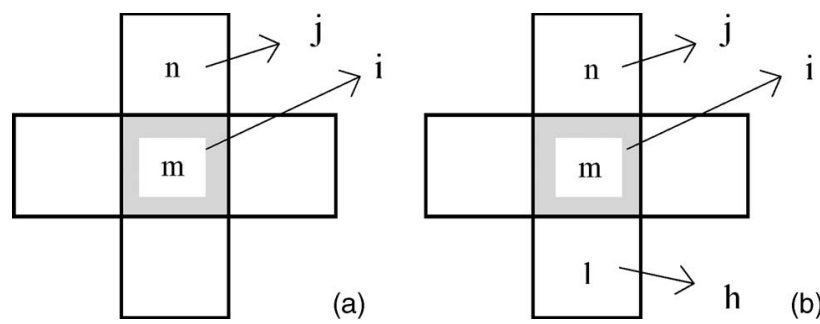

Fig. 1. (a) Second-order neighborhood for pixel $m$. (b) Third-order neighborhood for pixel $m$.

where $Q_{m}^{k}\left(\omega_{i}\right)$ is called the neighborhood function. Let $\aleph_{m}$ be a neighborhood as in Fig. 1(a) [19]. With the new evidence obtained from $Q$, we modified this initial probability, which is known as the posterior probability.

The function $Q_{m}^{k}\left(\omega_{i}\right)$ can be defined as

$$
Q_{m}^{k}\left(\omega_{i}\right)=\sum_{n} \sum_{j} p_{m n}\left(\omega_{i} \mid \omega_{j}\right) p_{m}^{k}\left(\omega_{j}\right)
$$

where $p_{m n}\left(\omega_{i} \mid \omega_{j}\right)$ is the probability that pixel $m$ belongs to class $\omega_{i}$, given that $n$ is from class $\omega_{i}$.

\section{Context With a Third-Order Statistic PLR3}

In this paper, we extend PLR2 to a new label relaxation approach termed PLR3, in which we consider third-order statistics. Third-order statistics have been shown to be important in many other fields [22], [34], [35].

The matrix $p_{m n}\left(\omega_{i} \mid \omega_{j}\right)$ is constructed considering not just one neighboring pixel $n$ but two neighbor pixels $n$ and $l$, for the actual pixel $m$; in this case, the conditional matrix will have the form $p_{m n l}\left(\omega_{i} \mid \omega_{j}, \omega_{h}\right)$. This is a conditional probability of seeing class $\omega_{i}$ for pixel $m$ given the following configuration: Pixel $n$ has class $\omega_{j}$, and pixel $l$ has class $\omega_{h}$.

For example, if we consider that the two classes are in a vertical (V) disposition, as shown in Fig. 1(b), the neighborhood function will be given by

$$
Q_{m}^{k}\left(\omega_{i}\right)=\sum_{j} \sum_{h} p_{m n l}\left(\omega_{i} \mid \omega_{j}, \omega_{h}\right) p_{n l}\left(\omega_{j} \mid \omega_{h}\right) p_{l}^{k}\left(\omega_{h}\right)
$$

and the a posteriori probability will be given by the same expression as (6).

The algorithm described in this section was applied to a series of synthetic images and a real image. The methodology is explained in Section III, using the example of a synthetic image created with different directions.

\section{METHOD}

Two types of synthetic images $(204 \times 204$ pixels $)$ were used, one with horizontal $(\mathrm{H})$ and $\mathrm{V}$ stripes and the other with diagonal stripes, as shown in Fig. 2. The images had four bands; the reason for choosing this number was because we also planned to use real images with four bands. Only two classes were considered for the experiments in an effort to simulate the extraction of a feature against a background, such as roads or other cartographic linear features. The synthetic image classes were produced with a random generator, and each band showed normal distributions: N1 [(40, 46, 46, 46), (10, 10, 10, 10)]

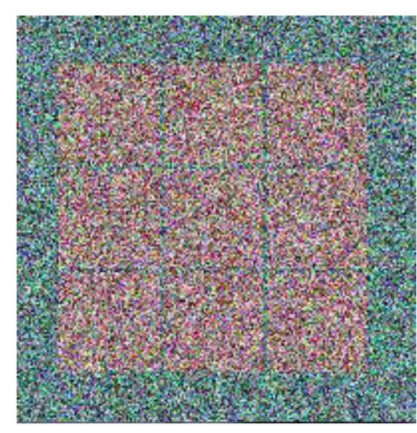

(a)

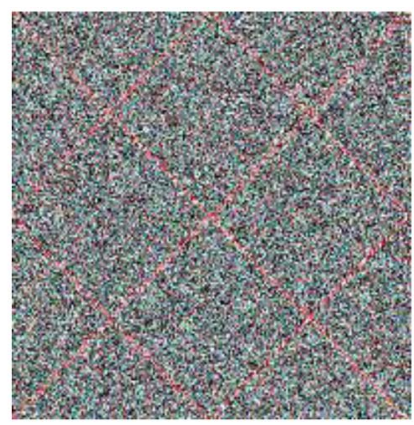

(b)
Fig. 2. Synthetic images (a) with $\mathrm{H}$ and $\mathrm{V}$ stripes and (b) with diagonal stripes.

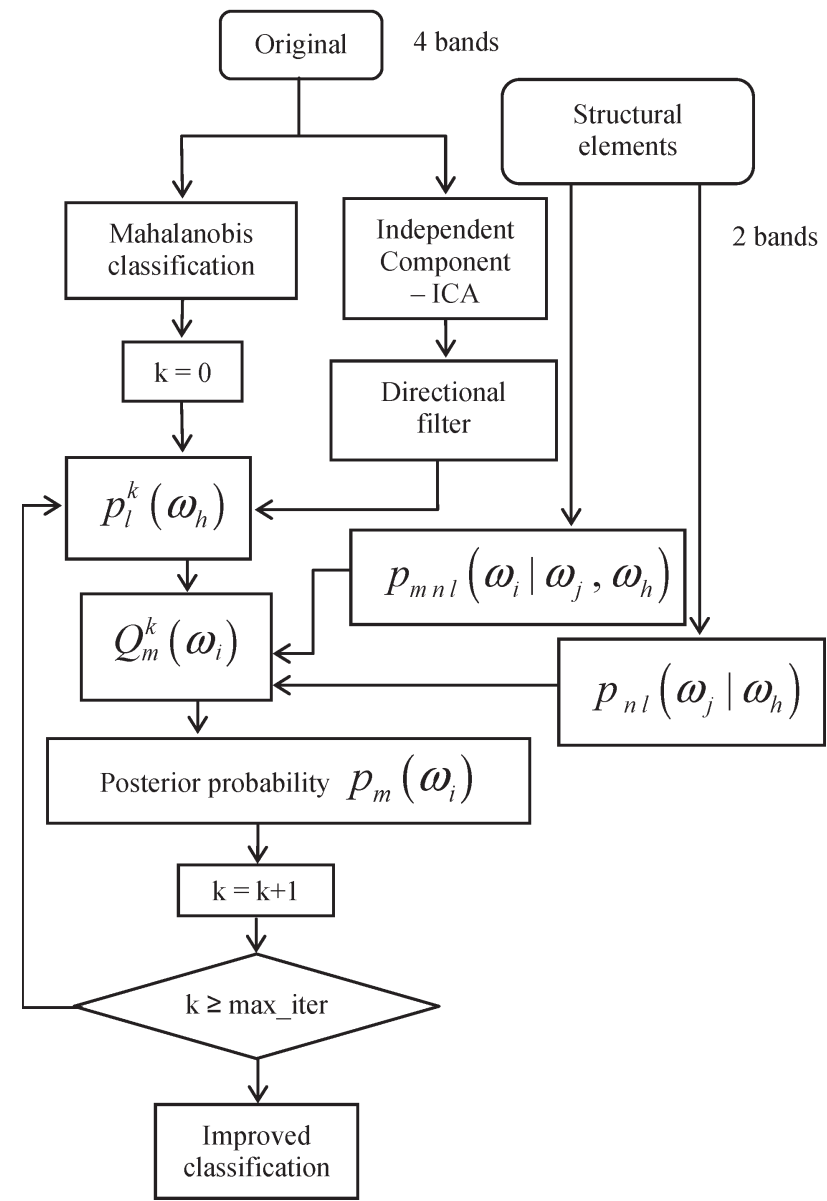

Fig. 3. Flowchart of the proposed method.

and $\mathrm{N} 2[(46,46,46,40),(10,10,10,10)]$ where the numbers represent different levels of gray for each band. Although only two classes were considered for the experiment, the method presented here would be valid for any number of classes.

Fig. 3 shows a flowchart of the proposed method. Entry of the algorithm is shown in the upper part. One of the inputs is the original image, which would be either a real or a synthetic image, as shown in Fig. 2(a) and (b).

The other input would be the structure to be detected in the original image. An example of this type of structure can be seen in Fig. 4. In Fig. 4(a), we look for $\mathrm{H}$ and $\mathrm{V}$ structures, and in Fig. 4(b), we look for principal (D1) and secondary (D2) diagonals. 


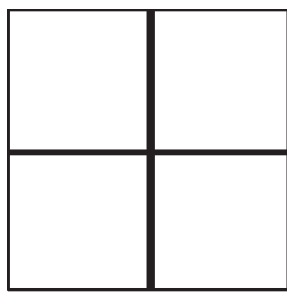

(a)

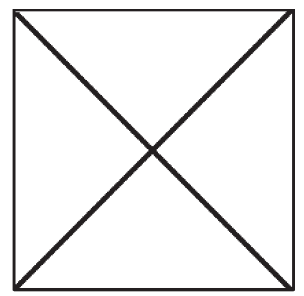

(b)
Fig. 4. Structural elements to be detected: (a) shows $\mathrm{H}$ and $\mathrm{V}$ structural elements, and (b) shows structural diagonals D1 and D2.

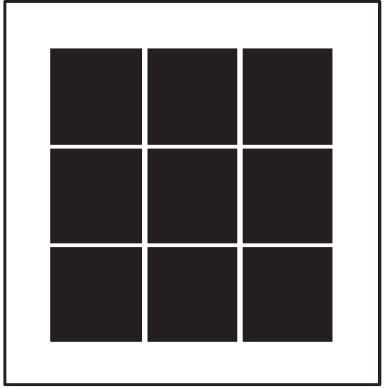

(a)

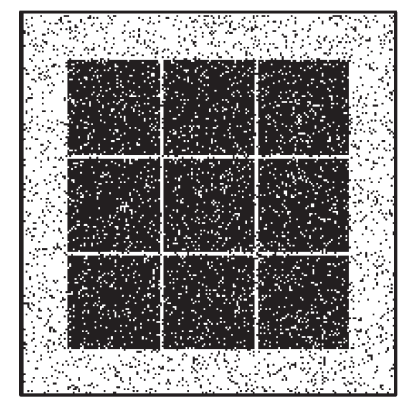

(c)

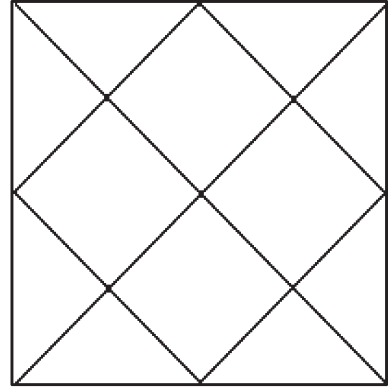

(b)

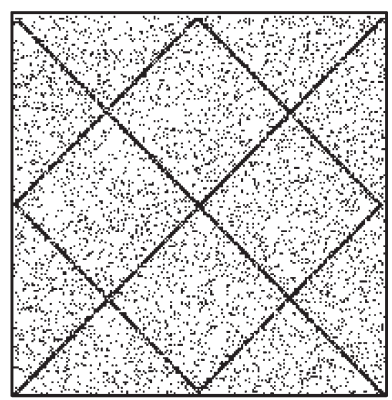

(d)
Fig. 5. (a) and (b) Ground truth. (c) and (d) Mahalanobis classification.

These structural elements are necessary to calculate the probabilities $p_{m n}\left(\omega_{i} \mid \omega_{j}\right)$ and $p_{m n i}\left(\omega_{i} \mid \omega_{j}, \omega_{h}\right)$ and to determine the neighborhood function of (8). It also allows the proportions of the two classes to be determined depending on the directions, as shown in Fig. 4. For the PLR2 method, a pair of pixels is taken, as shown in Fig. 1(a), while in the PLR3 method, triples of pixels are considered, as shown in Fig. 1(b). These triples are examined only for linear structures in all directions $(\mathrm{V}, \mathrm{H}$, and D1 and D2), and so consequently, the method preserves such linear features in the postclassification process.

The next step in the procedure is supervised classification of the images shown in Fig. 2 using training fields. In our case, as stated in the previous section, a Mahalanobis classification distance was used in both images, using $10 \%$ of the groundtruth images as a training set [Fig. 5(a) and (b)]. The results of the classification are shown in Fig. 5(c) and (d).

In order to determine the direction in which the algorithm should be applied ( $\mathrm{H}$ or $\mathrm{V}$, or diagonal $\mathrm{D} 1$ or $\mathrm{D} 2)$ in the current pixel, first, an independent component analysis (ICA) was carried out [36].

It is assumed that the reflectance of a pixel is a combination of the reflectances of endmember spectra in the area covered by that pixel; this mixture can be considered as the result of the linear combinations of these endmembers within the

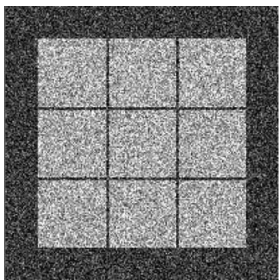

(a)

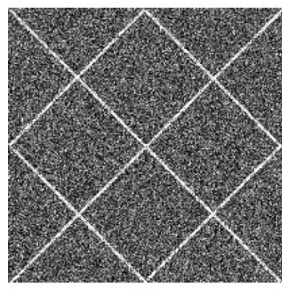

(c)

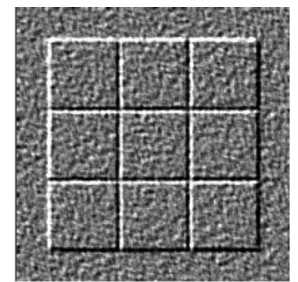

(b)

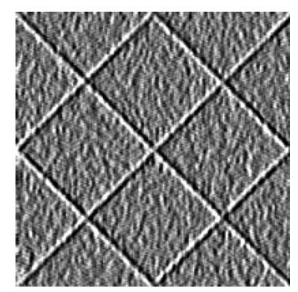

(d)
Fig. 6. (a) and (c) show the ICA H-V and D1-D2. (b) and (d) show the directional filter applied to the ICA analysis.

pixel [37]. Several authors have investigated the application of ICA methods to the analysis of remote sensing multispectral images [38]-[41]. ICA is defined as representing the pixel spectra by the linear combination of statistically independent components; since two classes are considered, two components were calculated for ICA. The features from the two classes behave to some extent like two different sources. For all cases of the synthetic and the real images, the first ICA band provided good differentiation between the features. Furthermore, a directional filter was used on the first band of the ICA to enhance identification of the adjacent pixel properties in all directions. The direction filter consisted of a $3 \times 3$ pixel window that studies the whole image, applying a higher weighting in a given direction $\left(0^{\circ}\right.$ and $90^{\circ}$ in the $\mathrm{H}$ and $\mathrm{V}$ and $45^{\circ}$ and $315^{\circ}$ in the $\mathrm{D} 1$ and D2). Fig. 6(a) and (c) shows the ICA images that contain edge information; Fig. 6(b) and (d) shows the directional filter applied to the ICA in order to enhance the directions.

The information from Fig. 6(b) and (d) is used to decide what values of $p_{m n l}\left(\omega_{i} \mid \omega_{j}, \omega_{h}\right)$ and $p_{n l}\left(\omega_{j} \mid \omega_{h}\right)$ should be used for the $\mathrm{H}, \mathrm{V}, \mathrm{D} 1$, or D2 directions, in each pixel. In the actual pixel, the direction is obtained by exploring the neighborhood pixels shown in Fig. 6(b) and (d). This information is also utilized in calculating $p_{l}^{k}\left(\omega_{h}\right)$, which updates the posterior probability in each iteration $k$.

The classifications are evaluated using a confusion matrix: accuracy and kappa coefficient with its p-value and 95\% confidence interval.

\section{RESUlts AND Discussion}

Fig. 7(a) shows the results of classifying the synthetic image shown in Fig. 2(a) by Mahalanobis distance, using $10 \%$ of the ground truth, shown in Fig. 5(a), as a training sample. Fig. 7(b)-(d) shows the postprocessing of the Mahalanobis classification. For Fig. 7(b), a Majority filter with a $3 \times 3$ window was applied, Fig. 7(c) was obtained with a PLR (PLR2), as explained by Richards and Jia [19], with three iterations, and in Fig. 7(d), the proposed PLR3 method was applied, also with three iterations. The key difference between PLR2 and PLR3 is that the former used the statistical distribution of pairs of 


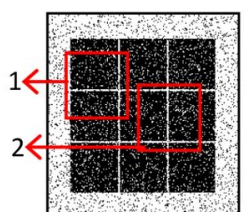

(a)

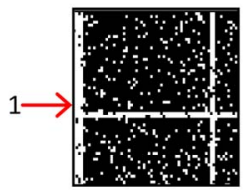

(e)

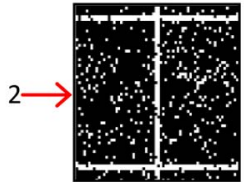

(i)

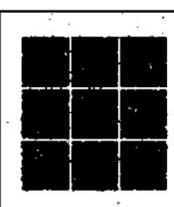

(b)

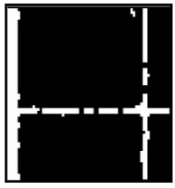

(f)

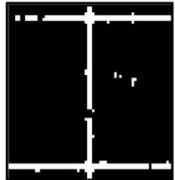

(j)

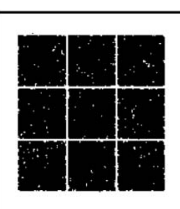

(c)

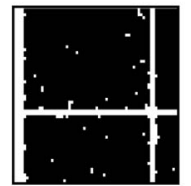

(g)

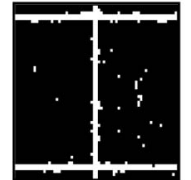

(k)

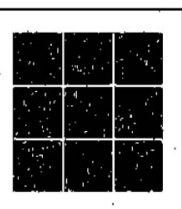

(d)

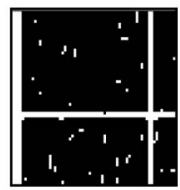

(h)

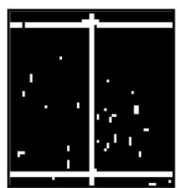

(I)
Fig. 7. Postprocessing of the (a) Mahalanobis classification with (b) Majority filter, (c) PLR2, and (d) PLR3 for the image with $\mathrm{H}$ and V structures. The details in (f)-(h) show the differences in the postprocessing algorithms. The white lines in (h), which correspond to PLR3, are better defined than the same lines in (f) (Majority) and (g) (PLR2). Similar results can be seen in the details of (j)-(l).

TABLE I

Confusion Matrices for Mahalanobis, Majority, PLR2, And PLR3 FOR H AND V STRUCTURES FOR THE SyNThETIC IMAGE IN Fig. 7

\begin{tabular}{|c|c|c|c|c|c|c|c|}
\hline \multicolumn{4}{|c|}{ MAHALANOBIS } & \multicolumn{4}{|c|}{ MAJORITY } \\
\hline & Black & White & Total & & Black & White & Total \\
\hline Black & 20573 & 1588 & 22161 & Black & 22623 & 156 & 22779 \\
\hline White & 2228 & 14504 & 16732 & White & 178 & 15936 & 16114 \\
\hline Total & 22801 & 16092 & 38893 & Total & 22801 & 16092 & 38893 \\
\hline \multicolumn{4}{|c|}{$(35077 / 38893) 90.19 \%$} & \multicolumn{4}{|c|}{$(38559 / 38893) 99.14 \%$} \\
\hline \multicolumn{4}{|c|}{$\begin{array}{c}\text { Kappa coefficient }=0.799 \\
\text { pvalue }<0.001\end{array}$} & \multicolumn{4}{|c|}{$\begin{array}{c}\text { Kappa coefficient }=0.982 \\
\text { pvalue }<0.001\end{array}$} \\
\hline \multicolumn{4}{|c|}{ CI 95\% Kappa $(0.793,0.805)$} & \multicolumn{4}{|c|}{ CI 95\% Kappa $(0.980,0.984)$} \\
\hline
\end{tabular}

\begin{tabular}{|c|c|c|c|c|c|c|c|}
\hline \multicolumn{4}{|c|}{ PLR2 } & \multicolumn{4}{|c|}{ PLR3 } \\
\hline & Black & White & Total & & Black & White & Total \\
\hline Black & 22291 & 3 & 22294 & Black & 22363 & 31 & 22394 \\
\hline White & 510 & 16089 & 16599 & White & 438 & 16061 & 16499 \\
\hline Total & 22801 & 16092 & 38893 & Total & 22801 & 16092 & 38893 \\
\hline \multicolumn{4}{|c|}{$(38380 / 38893) 98.68 \%$} & \multicolumn{4}{|c|}{$(38424 / 38893) 98.79 \%$} \\
\hline \multicolumn{4}{|c|}{$\begin{array}{c}\text { Kappa coefficient }=0.973, \\
\text { pvalue }<0.001\end{array}$} & \multicolumn{4}{|c|}{$\begin{array}{c}\text { Kappa coefficient }=0.975, \\
\text { pvalue }<0.001\end{array}$} \\
\hline \multicolumn{4}{|c|}{ CI 95\% Kappa $(0.971,0.975)$} & \multicolumn{4}{|c|}{ CI 95\% Kappa $(0.973,0.977)$} \\
\hline
\end{tabular}

pixels, while the latter used triples of pixels. It is observed that all three methods reduced the noise of the initial Mahalanobis classification, particularly the Majority filter in Fig. 7(b); however, PLR2 and PLR3 better delineate the linear entities, as can be seen in the details hereinafter, in Fig. 7(e)-(l). The images in Fig. 7(e) and (i) are details of the Mahalanobis classification shown in Fig. 7(a); in Fig. 7(f) and (j), corresponding to the Majority filter, black pixels invade white lines; in Fig. $7(\mathrm{~g})$ and $(\mathrm{k})$, corresponding to PLR2, white pixels occupy the black edges; Fig. 7(h) and (1), corresponding to PLR3, produces the best results in depicting the white lines.

To complement this visual analysis, we performed a numerical analysis in which the classification was evaluated using a confusion matrix of the full ground-truth data set [Fig. 5(a) and (b)]. Table I shows the results of the confusion matrices for Mahalanobis and the three postclassification methods.

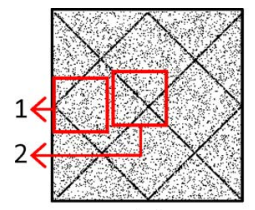

(a)

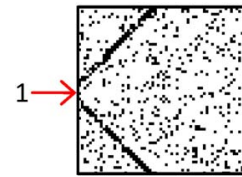

(e)

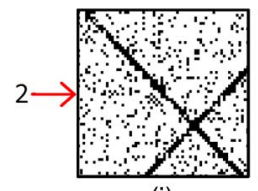

(i)

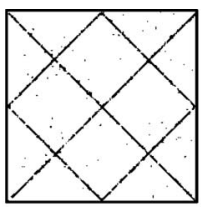

(b)

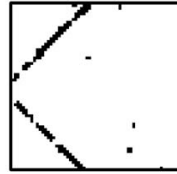

(f)

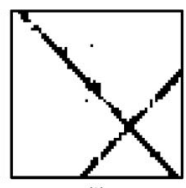

(j)

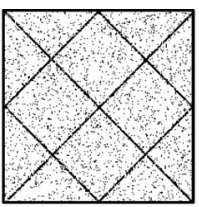

(c)

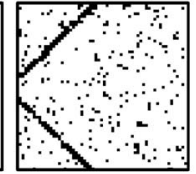

(g)

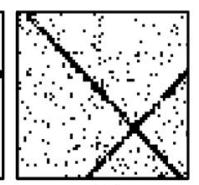

(k)

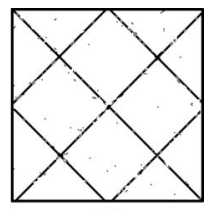

(d)

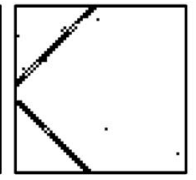

(h)

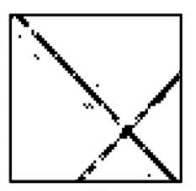

(I)
Fig. 8. Postprocessing of the (a) Mahalanobis classification with (b) Majority filter, (c) PLR2, and (d) PLR3 for the image with diagonals D1 and D2. Details in (e)-(1) show the differences in the postprocessing algorithms. (f) and (j) show two details of the Majority filter, $(\mathrm{g})$ and $(\mathrm{k})$ represent the results of the PLR2 algorithm, and (h) and (1) show the performance of the proposed algorithm PLR3.

TABLE II

Confusion Matrices for Mahalanobis, Majority, PLR2, And PLR3 FOR D1 AND D2 STRUCTURES FOR SYNTHETIC IMAGE IN FIG. 8

\begin{tabular}{|c|c|c|c|c|c|c|c|}
\hline \multicolumn{4}{|c|}{ MAHALANOBIS } & \multicolumn{4}{|c|}{ MAJORITY } \\
\hline & Black & White & Total & & Black & White & Total \\
\hline Black & 1827 & 4628 & 6455 & Black & 1855 & 314 & 2169 \\
\hline White & 280 & 31109 & 31389 & White & 252 & 35423 & 35675 \\
\hline Total & 2107 & 35737 & 37844 & Total & 2107 & 35737 & 37844 \\
\hline \multicolumn{4}{|c|}{$(32936 / 37844) 87.03 \%$} & \multicolumn{4}{|c|}{$(37278 / 37844) 98.50 \%$} \\
\hline \multicolumn{4}{|c|}{$\begin{array}{c}\text { Kappa coefficient }=0.374, \\
\text { pvalue }<0.001\end{array}$} & \multicolumn{4}{|c|}{$\begin{array}{c}\text { Kappa coefficient }=0.860 \\
\text { pvalue }<0.001\end{array}$} \\
\hline \multicolumn{4}{|c|}{ CI 95\% Kappa $(0.360,0.388)$} & \multicolumn{4}{|c|}{ CI 95\% Kappa $(0.848,0.872)$} \\
\hline
\end{tabular}

\begin{tabular}{|c|c|c|c|c|c|c|c|}
\hline \multicolumn{4}{|c|}{ PLR2 } & \multicolumn{4}{|c|}{ PLR3 } \\
\hline & Black & White & Total & & Black & White & Total \\
\hline Black & 1876 & 2554 & 4430 & Black & 1881 & 107 & 1988 \\
\hline White & 231 & 33183 & 33414 & White & 226 & 35630 & 35856 \\
\hline Total & 2107 & 35737 & 37844 & Total & 2107 & 35737 & 37844 \\
\hline \multicolumn{4}{|c|}{$(35059 / 37844) 92.64 \%$} & \multicolumn{4}{|c|}{$(37511 / 37844) 99.12 \%$} \\
\hline \multicolumn{4}{|c|}{$\begin{array}{c}\text { Kappa coefficient }=0.539 \\
\text { pvalue }<0.001\end{array}$} & \multicolumn{4}{|c|}{$\begin{array}{c}\text { Kappa coefficient }=0.914, \\
\text { pvalue }<0.001\end{array}$} \\
\hline \multicolumn{4}{|c|}{ CI 95\% Kappa $(0.523,0.555)$} & \multicolumn{4}{|c|}{ CI 95\% Kappa $(0.904,0.924)$} \\
\hline
\end{tabular}

Note that the Majority method provides the best accuracy (99.14\%) and kappa coefficient (0.982), with approximately 300 pixels misclassified; the PLR3 produced the second best accuracy (98.79\% and kappa coefficient 0.9752$)$ with almost 500 pixels incorrectly classified, but better defined linear features. Most of the decrease in PLR3 accuracy and kappa is because, for this case, the method, as well as the Majority method, does not clean noise; Majority reduced the noise but increased the misclassified pixels in the edges of linear structures. PLR2 was slightly less accurate (accuracy 98.68\% and kappa 0.973) than the PLR3 and Majority algorithms.

For all methods, the p-value and a 95\% confidence interval have been included. In all cases, the p-value was less than 0.001 , indicating that the classifications obtained are significant. Although the confidence intervals for PLR2 and PLR3 have no empty intersection, which means that the difference 


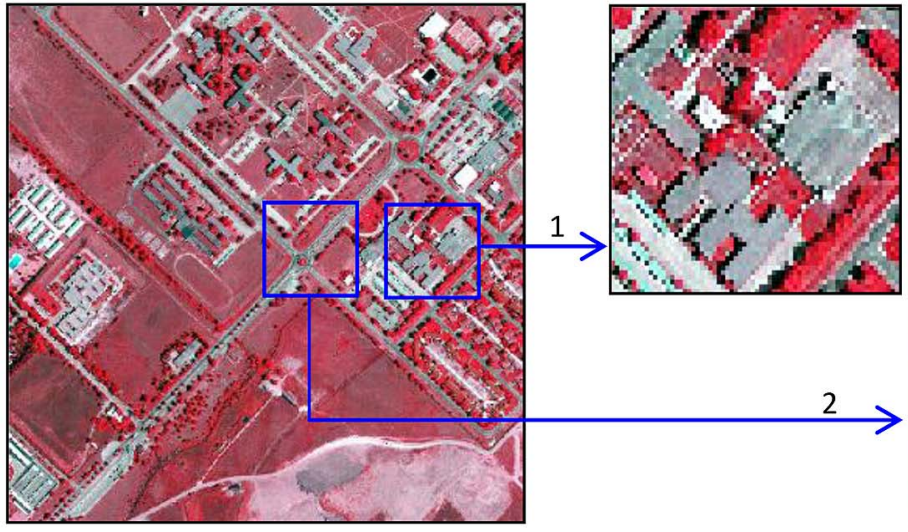

(a)

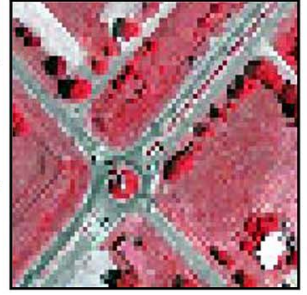

?

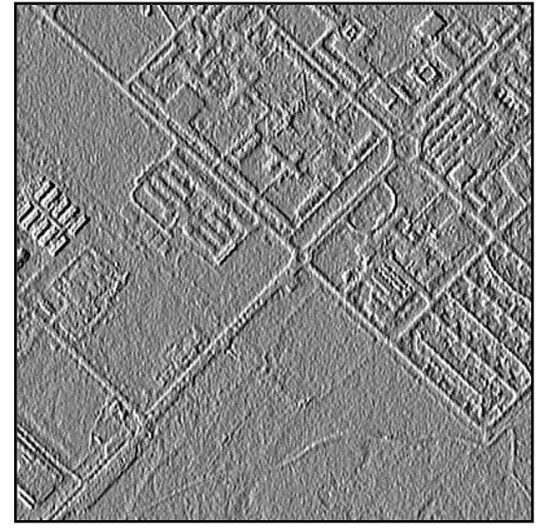

(b)

Fig. 9. (a) Aerial image of an area of Madrid with two details. Image (b) shows the first band of ICA with diagonal filters applied.

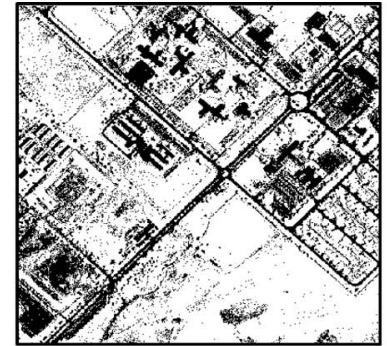

(a)

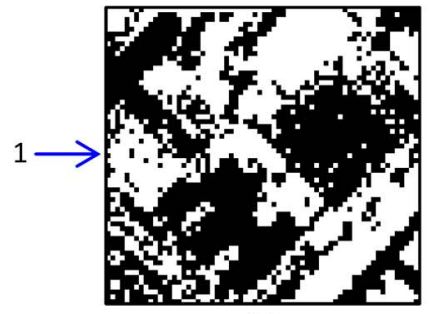

(e)

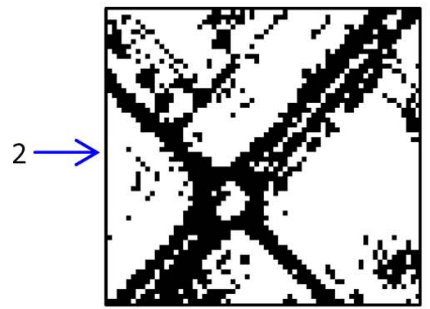

(i)

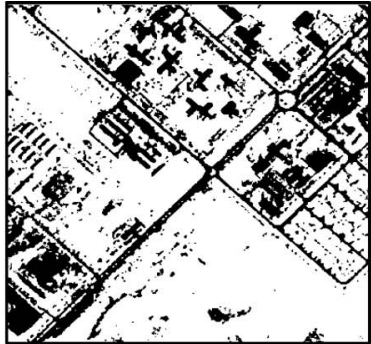

(b)

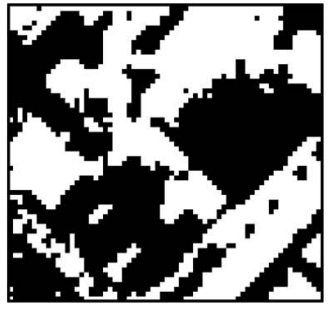

(f)

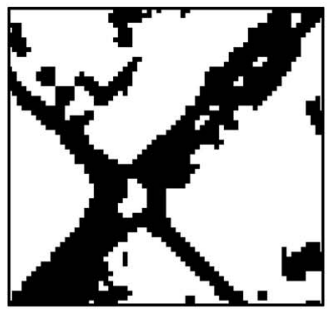

(j)

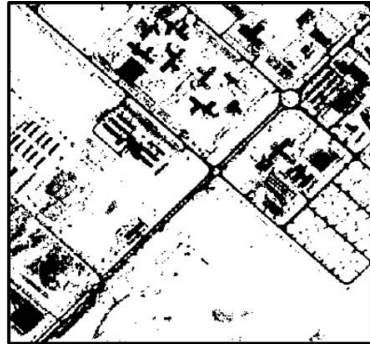

(c)

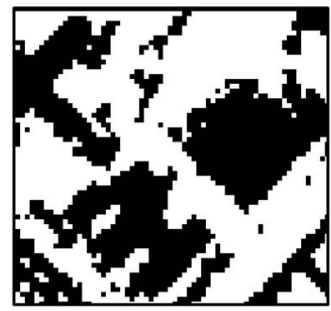

(g)

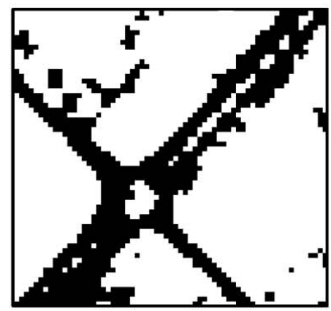

(k)

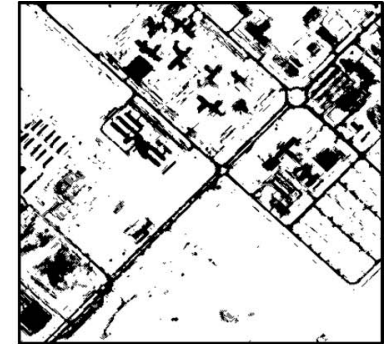

(d)

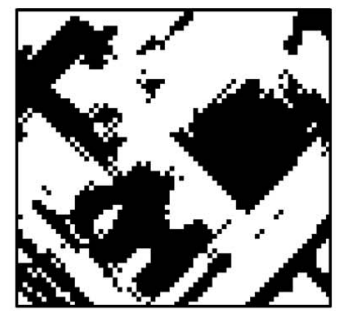

(h)

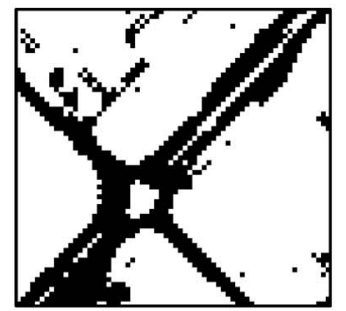

(I)

Fig. 10. Postprocessing of the (a) Mahalanobis classification with (b) Majority filter, (c) PLR2, and (d) PLR3 for the aerial image. Details in (e)-(1) show the differences in the postprocessing algorithms. (f) and (j) show two details of the Majority filter, (g) and (k) represent the results of the PLR2 algorithm, and (h) and (1) show the performance of the proposed algorithm.

between methods is statistically nonsignificant (as can be seen in Table I), the visual evaluation [Fig. 7(g) compared with Fig. 7(h) and Fig. 7(k) compared with Fig. 7(1)] shows that the edges are better represented by PLR3 than by PLR2.

We conducted a similar study for diagonal directions. The Mahalanobis classification and the three postclassification methods for Fig. 2(b) image with some details are shown in Fig. 8. Following the order of the aforementioned synthetic image, Fig. 8(a) corresponds to Mahalanobis classification, Fig. 8(b) corresponds to Majority filter, Fig. 8(c) corresponds to PLR2, and Fig. 8(d) corresponds to PLR3.
The worst performance of the three postclassification techniques in this case was the PLR2 method, which produced a lot of noise and poor determination of the linear features [Fig. 8(c)]. This is due to the shape of the neighborhood considered for PLR2, as this method studies the images in only $\mathrm{H}$ and $\mathrm{V}$ directions. The number of noisy pixels was similar in the Majority and PLR3 results; however, as with the previous synthetic image, PLR3 defined the linear features more accurately, as seen by comparing Fig. 8(b) and (d). Two details [Fig. 8(e)-(h) and (i)-(l)] have been provided to better see this idea. It can be observed that Fig. 8(h) and (l) are the 
TABLE III

CONFUsion MATRices For MAHALANOBis, MAJORITy, PLR2, AND PLR3 FOR D1 AND D2 STRUCTURES FOR THE AERIAL IMAGE IN Fig. 9

\begin{tabular}{|c|c|c|c|c|c|c|c|}
\hline \multicolumn{4}{|c|}{ MAHALANOBIS } & \multicolumn{4}{|c|}{ MAJORITY } \\
\hline & Black & White & Total & & Black & White & Total \\
\hline Black & 689 & 280 & 969 & Black & 728 & 236 & 964 \\
\hline White & 67 & 1612 & 1679 & White & 28 & 1656 & 1684 \\
\hline Total & 756 & 1892 & 2648 & Total & 756 & 1892 & 2648 \\
\hline \multicolumn{4}{|c|}{$(2301 / 2648) 86.8958 \%$} & \multicolumn{4}{|c|}{$(2384 / 2648) 90.0302 \%$} \\
\hline \multicolumn{4}{|c|}{$\begin{array}{c}\text { Kappa coefficient }=0,704, \\
\text { pvalue }<0.001\end{array}$} & \multicolumn{4}{|c|}{$\begin{array}{c}\text { Kappa coefficient }=0.774 \\
\text { pvalue }<0.001\end{array}$} \\
\hline \multicolumn{4}{|c|}{ CI 95\% Kappa $(0.675,0.733)$} & \multicolumn{4}{|c|}{ CI 95\% Kappa $(0.749,0.799)$} \\
\hline
\end{tabular}

\begin{tabular}{|c|c|c|c|c|c|c|c|}
\hline \multicolumn{4}{|c|}{ PLR2 } & \multicolumn{4}{|c|}{ PLR3 } \\
\hline & Black & White & Total & & Black & White & Total \\
\hline Black & 703 & 126 & 829 & Black & 726 & 141 & 867 \\
\hline White & 53 & 1766 & 1819 & White & 30 & 1751 & 1781 \\
\hline Total & 756 & 1892 & 2648 & Total & 756 & 1892 & 2648 \\
\hline \multicolumn{4}{|c|}{$(2469 / 2648) 93.2402 \%$} & \multicolumn{4}{|c|}{$(2477 / 2648) 93.5423 \%$} \\
\hline \multicolumn{4}{|c|}{$\begin{array}{c}\text { Kappa coefficient }=0.839 \\
\text { pvalue }<0.001\end{array}$} & \multicolumn{4}{|c|}{$\begin{array}{c}\text { Kappa coefficient }=0.848, \\
\text { pvalue }<0.001\end{array}$} \\
\hline \multicolumn{4}{|c|}{ CI 95\% Kappa $(0.815,0.863)$} & \multicolumn{4}{|c|}{ CI 95\% Kappa $(0.826,0.870)$} \\
\hline
\end{tabular}

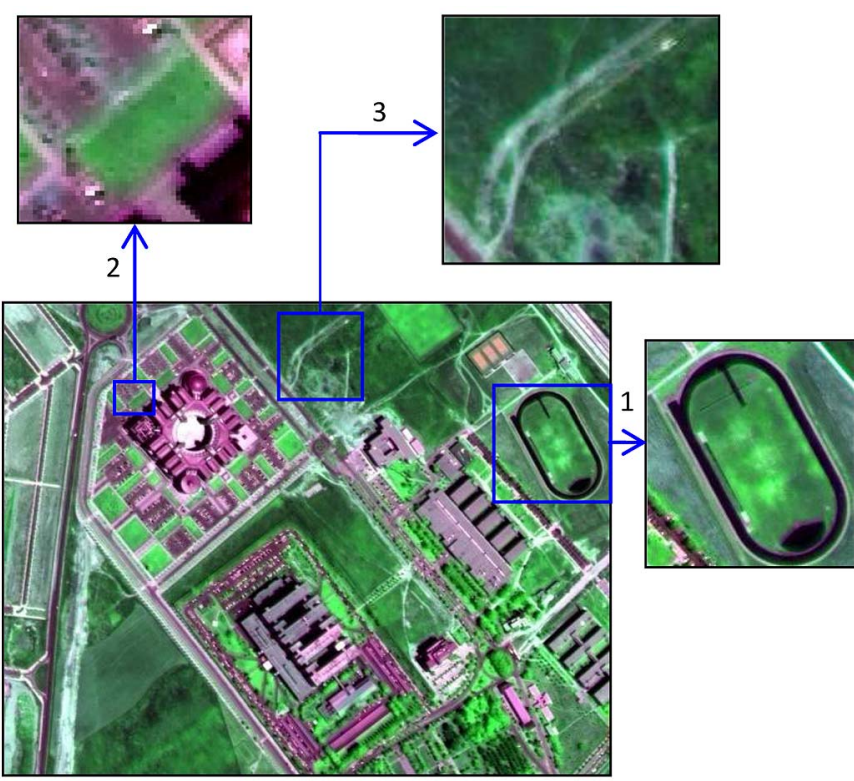

(a)

Fig. 11. Image (a) is a satellite image (Ikonos) of the Alcalá de Henares university campus (Madrid) with three details 1, 2, and 3. The bands for visualization with red, green, and blue are infrared, blue, and green, respectively.

best postprocessing classifications among the three methods, removing noise and preserving linear features, as confirmed by numerical analysis in Table II.

In this case, PLR3 provides the best accuracy $(99.12 \%)$ and kappa (0.9140), while Majority provides an accuracy of $98.50 \%$ with a kappa of 0.860 . As in Table I, PLR3 produced more noise in homogeneous regions than Majority but worked better at the edges. PLR3 and Majority present significant differences in the kappa coefficient because their confidence interval at the $95 \%$ level had an empty intersection.

Fig. 9 shows an aerial image $(400 \times 400$ pixels $)$ of Alcalá de Henares, Madrid, Spain, taken in summer 2010. This image was taken with a Leica ADS40 SH52 sensor, with a spatial resolution of $0.5 \mathrm{~m}$ and four spectral bands (red, green, blue, and near infrared). The two images on the right are the selected details. In this case, the diagonal neighborhood

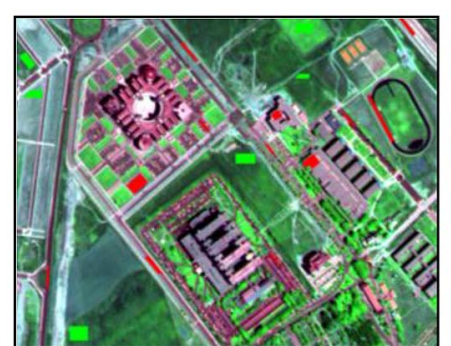

(a)

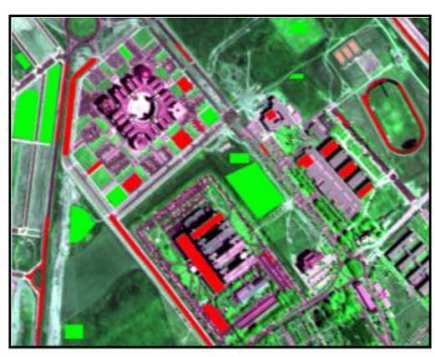

(b)

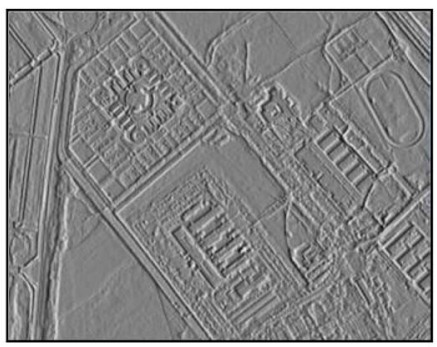

(c)

Fig. 12. Panels (a) and (b) show the training and evaluation sets, respectively, for Fig. 11 satellite image. Panel (c) shows the first band of ICA analysis with diagonal filters applied.

TABLE IV

CONFUSION MATRICES FOR MAHALANOBIS, MAJORITY, PLR2, AND PLR3 FOR D1 AND D2 STRUCTURES FOR IKONOS IMAGE IN FIG. 11

\begin{tabular}{|c|c|c|c|c|c|c|c|}
\hline \multicolumn{4}{|c|}{ MAHALANOBIS } & \multicolumn{4}{|c|}{ MAJORITY } \\
\hline & Black & White & Total & & Black & White & Total \\
\hline Black & 22900 & 116 & 23016 & Black & 23017 & 101 & 23118 \\
\hline White & 1412 & 25209 & 26621 & White & 1295 & 25224 & 26519 \\
\hline Total & 24312 & 25325 & 49637 & Total & 24312 & 25325 & 49637 \\
\hline \multicolumn{4}{|c|}{$(48109 / 49637) 96.9217 \%$} & \multicolumn{4}{|c|}{$(48241 / 49637) 97.1876 \%$} \\
\hline \multicolumn{4}{|c|}{$\begin{array}{c}\text { Kappa coefficient }=0.938, \\
\text { pvalue }<0.001\end{array}$} & \multicolumn{4}{|c|}{$\begin{array}{c}\text { Kappa coefficient }=0.944, \\
\text { pvalue }<0.001\end{array}$} \\
\hline \multicolumn{4}{|c|}{ CI 95\% Kappa $(0.934,0.942)$} & \multicolumn{4}{|c|}{ CI 95\% Kappa $(0.942,0.946)$} \\
\hline
\end{tabular}

\begin{tabular}{|c|c|c|c|c|c|c|c|}
\hline \multicolumn{4}{|c|}{ PLR2 } & \multicolumn{4}{|c|}{ PLR3 } \\
\hline & Black & White & Total & & Black & White & Total \\
\hline Black & 22904 & 91 & 22995 & Black & 24010 & 534 & 24544 \\
\hline White & 1408 & 25234 & 26642 & White & 302 & 24791 & 25093 \\
\hline Total & 24312 & 25325 & 49637 & Total & 24312 & 25325 & 49637 \\
\hline \multicolumn{4}{|c|}{$(48138 / 49637) 96.9801 \%$} & \multicolumn{4}{|c|}{$(48801 / 49637) 98.3158 \%$} \\
\hline \multicolumn{4}{|c|}{$\begin{array}{c}\text { Kappa coefficient }=0.940, \\
\text { pvalue }<0.001\end{array}$} & \multicolumn{4}{|c|}{$\begin{array}{c}\text { Kappa coefficient }=0.970, \\
\text { pvalue }<0.001\end{array}$} \\
\hline \multicolumn{4}{|c|}{ CI 95\% Kappa $(0.936,0.944)$} & \multicolumn{4}{|c|}{ CI 95\% Kappa $(0.968,0.972)$} \\
\hline
\end{tabular}

system was used because of the diagonal appearance of the man-made and natural features of the image. The result of applying the directional filter to the first component of the ICA is shown in Fig. 9(b), which shows how the linear structures are enhanced.

The classification and postprocessing are shown in Fig. 10; as with the synthetic images, the first row shows Mahalanobis (a), Majority (b), PLR2 (c), and PLR3 (d) applied to Fig. 9(a). It can be seen that the three postprocessing methods removed most of the noise from the Mahalanobis classification, but boundaries are represented very differently between the studied methods; at this level, it can also be seen that PLR3 best conserves the linear features. To appreciate this characteristic, we show the two details of Fig. 9(a): Number 1 shows a region with buildings in a diagonal disposition, and number 2 shows the diagonal intersection of two roads. 


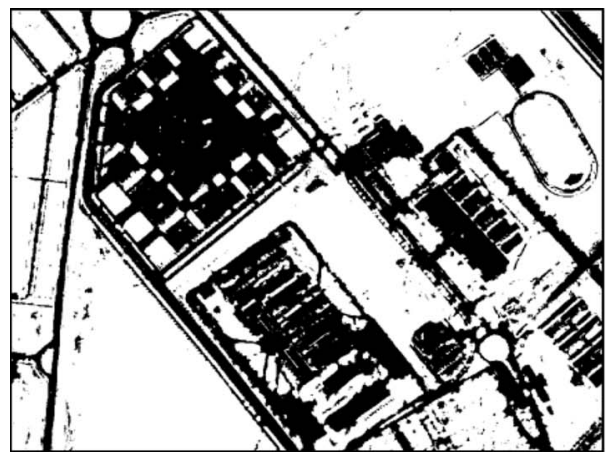

(a)

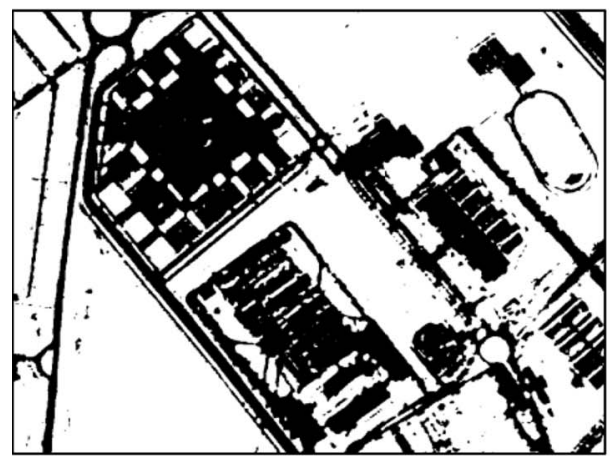

(c)

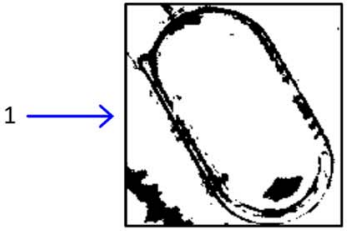

(e)

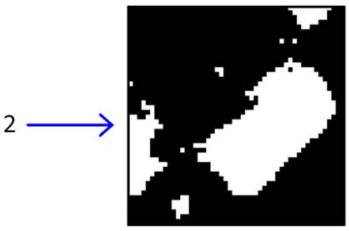

(i)

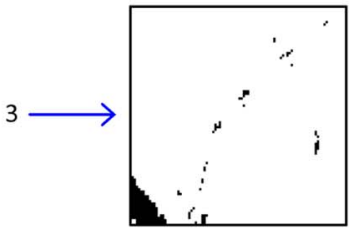

(m)

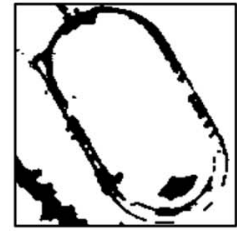

(f)

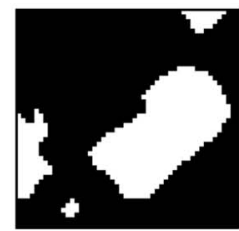

(j)

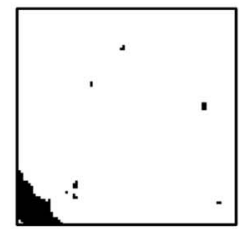

(n)

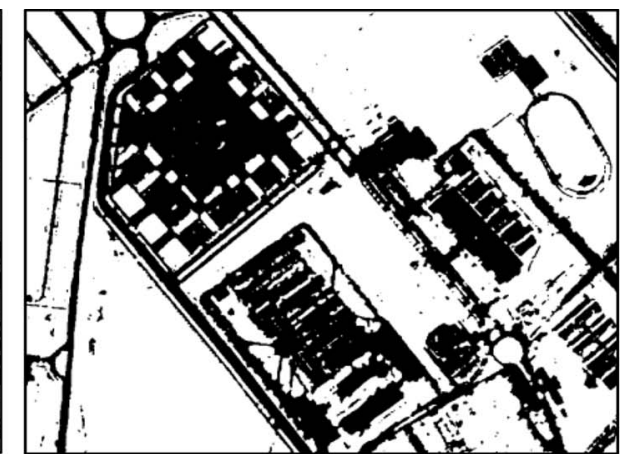

(b)

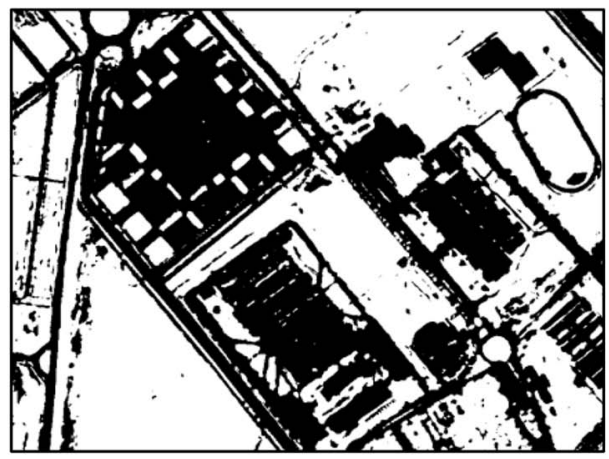

(d)

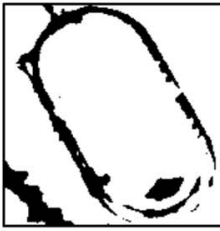

(g)

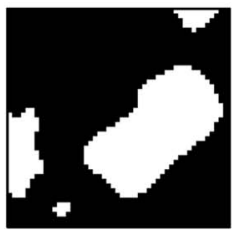

(k)

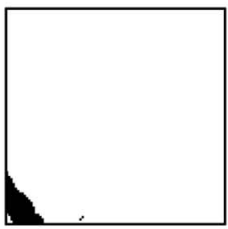

(o)

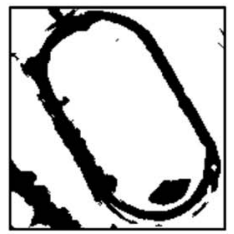

(h)

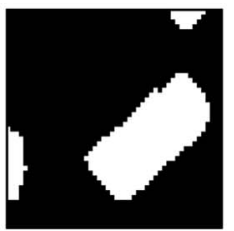

(I)

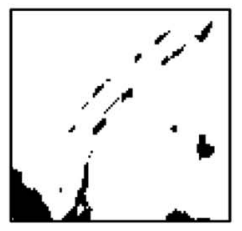

(p)

Fig. 13. Postprocessing of (a) the Mahalanobis classification with (b) Majority filter, (c) PLR2, and (d) PLR3 of the real image in Fig. 12. The other images show the results of the tested algorithms in three scenes of the image. First column [(e), (i), and (m)] shows the Mahalanobis classification, second column shows the Majority filter, third column shows the PLR2, and fourth column shows the proposed algorithm PLR3.

In the first detail, note how in Fig. 10(h), corresponding to PLR3, the large building at the center right is better delineated than in Fig. 10(e)-(g), corresponding to Mahalanobis, Majority, and PLR2, respectively. In the second detail, note how in Fig. 10(1), corresponding to PLR3, the median road is detected more clearly than in Fig. 10(i)-(k).

Apart from the visual evaluation, Table III shows the confusion matrices and p-values, which are superior for the PLR3 method although, for this case, the comparison between PLR3 and PLR2 is significant to the $95 \%$ level.
Fig. 11 shows another real image, which is this time a satellite image from the Ikonos sensor; this sensor takes images in four spectral bands with different spatial resolutions $(1 \mathrm{~m}$ in the panchromatic mode and $4 \mathrm{~m}$ in the multispectral mode) from an average altitude of $681 \mathrm{~km}$ (revisit time is approximately three days). The image used here is a pansharpened image [42] of $1 \mathrm{~m}$ resolution and a size of $900 \times 700 \mathrm{~m}$, depicting part of Alcala university campus, Madrid.

A training set was selected from Fig. 11(a), as shown in Fig. 12(a). In remote sensing classification with only two 
classes, the most commonly chosen classes are man-made and natural areas. Training areas are shown in red for man-made areas and bright green for natural objects, which are mostly vegetation with some areas of bare soil. The training areas in Fig. 12(a) were extended to produce the evaluation set seen in Fig. 12(b), so different pixels are used for evaluation and training. Fig. 12(c) shows the image produced by applying the directional filter to the first ICA component from Fig. 11(a). The results of the classification are presented in Table IV. All three postprocessing method have an accuracy and kappa coefficient superior to the initial classification by Mahalanobis distance, with the PLR3 method producing the best results. The high confidence interval and kappa value demonstrate that the PRL3 method is significantly superior to the Majority and PLR2 methods.

Mahalanobis classification of the image in Fig. 11(a) is shown in Fig. 13(a), demonstrating that most man-made areas were classified correctly. Fig. 13(b)-(d) shows the postclassification of Fig. 13(a) with Majority, PLR2, and PLR3, respectively. Note that the tennis courts and athletics track in the upper right part of Fig. 11(a) have synthetic surfaces, so they have been correctly assigned to the man-made class.

For a visual discussion of the results, three details have been selected from the classified satellite image to evaluate the goodness of the algorithm. The first detail [Fig. 11(a)-1] shows the athletics track. In Fig. 13(e), the results of the Mahalanobis classifications are shown; the Majority postprocessed image is shown in Fig. 13(f), showing some improvement such as reduced noise. Furthermore, Fig. 13(g)-(h) corresponds to PLR2 and PLR3, respectively, and shows further improvement over Majority, with reduced noise and more clearly delineated track.

The second detail [Fig. 11(a)-2] corresponds to a natural area, such as a lawn; the Mahalanobis classification of this element is shown in Fig. 13(i); the postclassification images with Majority, PLR2, and PLR3 are shown in Fig. 13(j)-(l), respectively. All three improve on the Mahalanobis classification, but the proposed PLR3 method is superior to the other methods in preserving linear structures. Finally, the third detail [Fig. 11(a)-3] corresponds to a major and a minor road; the Mahalanobis classification is shown in Fig. 13(m). The Majority [Fig. 13(n)] and PLR2 [Fig. 13(o)] blindly clean the noise, removing the minor road, while PLR3 preserved part of the diagonal linear structures, keeping and extending some of the lines delineating the minor road [Fig. 13(p)].

\section{CONCLusion}

This paper has examined the use of a postclassification method to improve the supervised classification of linear structures. We compared the behavior of three methods: two preexisting methods, Majority and PLR2, and a proposed PLR3 method, which extends PLR2 by studying groups of three pixels in the current pixel neighborhood rather than pairs.

In general contextual postclassification, it is important to remove the noise of an initial classification. Existing methods are useful in homogeneous regions, but when boundaries need to be enhanced, many problems occur with misclassified pixels at the edges of the classification regions. The goal of the proposed PLR3 method, in addition to removing classification noise, is to correctly differentiate linear boundaries between classes.

The performance of the three methods was tested in four images: two synthetic images and two real images (an aerial and a satellite image). The use of synthetic images with full ground truth facilitated numerical analysis of the accuracy and kappa coefficient of the different methods. The use of real images was important to determine the potential of the proposed method for real situations.

The results showed that, in all cases, postclassification improved the accuracy of the initial classification by reducing image noise. The proposed PLR3 method defines linear structures better than the Majority and PLR2 methods, due to the inclusion of third-order statistics (in the sense of using triplets of neighborhood pixels) within the probabilistic likelihood calculation.

The proposed PLR3 method was used to extract linear features such as roads, sports tracks, buildings, etc., from remotely sensed aerial and satellite images; the results showed that the proposed method was a good candidate for a postclassifier. There is no single best postprocessing technique for all cases because results depend on the application at hand. Therefore, the method presented here is useful when it is important to retain lines or edges.

\section{REFERENCES}

[1] T. Lillesand, R. Kiefer, and J. Chipman, Remote Sensing and Image Interpretation, 6th ed. New York: Wiley, 2008.

[2] L. D. Robertson and D. King, "Comparison of pixel- and object-based classification in land cover change mapping," Int. J. Remote Sens., vol. 32, no. 6, pp. 1505-1529, Mar. 2011

[3] H. R. Matinfar, F. Sarmadian, S. K. Alavi Panah, and R. J. Heck, "Comparisons of object-oriented and pixel-based classification of land use/land cover types based on Landsat 7, ETM+ spectral bands (case study: Arid region of Iran)," Amer-Eurasian J. Agriculture Environ. Sci., vol. 2, no. 4, pp. 448-456, 2007.

[4] I. Yildirim, O. K. Ersoy, and B. Yazgan, "Improvement of classification accuracy in remote sensing using morphological filter," Adv. Space Res., vol. 36, no. 5, pp. 1003-1006, 2005.

[5] R. L. Ketting and D. A. Landgrebe, "Classification of multispectral image data by extraction and classification of homogeneous objects," IEEE Trans. Geosci. Electron., vol. GE-14, no. 1, pp. 19-26, Jan. 1976.

[6] B. Solaiman, R. K. Koffi, M. C. Mouchot, and A. Hillion, "An information fusion method for multispectral image classification postprocessing," IEEE Trans. Geosci. Remote Sens., vol. 36, no. 2, pp. 395-406, Mar. 1998.

[7] J. Stuckens, P. R. Coppin, and M. E. Bauer, "Integrating contextual information with per-pixel classification for improved land cover classification," Remote Sens. Environ., vol. 71, no. 3, pp. 282-296, Mar. 2000.

[8] J. R. Eastman, IDRISI for Windows, User's Guide. Worcester, MA: Clark Univ., 1995.

[9] K. E. Kim, "Adaptive majority filtering for contextual classification of remote sensing data," Int. J. Remote Sens., vol. 17, no. 5, pp. 1083-1087, 1996.

[10] X. Jia and J. A. Richards, "Managing the spectral-spatial mix in context classification using Markov random fields," IEEE Geosci. Remote Sens. Lett., vol. 5, no. 2, pp. 311-314, Apr. 2008.

[11] A. H. S. Solberg, T. Taxt, and A. K. Jain, "A Markov random field model for classification of multisource satellite imagery," IEEE Trans. Geosci. Remote Sens., vol. 34, no. 1, pp. 100-113, Jan. 1996.

[12] A. H. S. Solberg, "Contextual data fusion applied to forest map revision," IEEE Trans. Geosci. Remote Sens., vol. 37, no. 3, pp. 1234-1243, May 1999.

[13] F. Melgani and S. B. Serpico, "A Markov random field approach to spatiotemporal contextual image classification," IEEE Trans. Geosci. Remote Sens., vol. 41, no. 11, pp. 2478-2487, Nov. 2003.

[14] D. Liu, M. Kelly, and P. Gong, "A spatial-temporal approach to monitoring forest disease spread using multi-temporal high spatial resolution imagery," Remote Sens. Environ., vol. 101, no. 2, pp. 167-180, 2006. 
[15] F. Melgani, B. A. R. Al Hashemy, and S. M. R. Taha, "An explicit fuzzy supervised classification method for multispectral remote sensing images," IEEE Trans. Geosci. Remote Sens., vol. 38, no. 1, pp. 287-295, Jan. 2000 .

[16] C. Benz Ursula, P. Hofmann, G. Willhauck, I. Lingenfelder, and M. Heynen, "Multi-resolution, object-oriented fuzzy analysis of remote sensing data for GIS-ready information," ISPRS J. Photogramm. Remote Sens., vol. 58, no. 3/4, pp. 239-258, Jan. 2004.

[17] S. W. Myint, P. Gober, A. Brazel, S. Grossman-Clarke, and Q. Weng, "Perpixel vs. object-based classification of urban land cover extraction using high spatial resolution imagery," Remote Sens. Environ., vol. 115, no. 5, pp. 1145-1161, 2011.

[18] S. Geman and D. Geman, "Stochastic relaxation, Gibbs distributions and Bayesian restoration of images," IEEE Trans. Pattern Anal. Mach. Intell., vol. PAMI-6, no. 6, pp. 721-741, Jun. 1984.

[19] J. A. Richards and X. Jia, Remote Sensing Digital Image Analysis, 4th ed. New York: Springer-Verlag, 2006.

[20] J. Stuckens, P. R. Coppin, and M. E. Bauer, "Integrating contextual information with per-pixel classification for improved land cover classification," Remote Sens. Environ., vol. 71, no. 3, pp. 282-296, Mar. 2000.

[21] S. Myeong, D. J. Nowak, P. F. Hopkins, and R. H. Brock, "Urban cover mapping using digital, high-spatial resolution aerial imagery," Urban Ecosyst., vol. 5, no. 4, pp. 243-256, Dec. 2001.

[22] D. J. Field, "Relations between the statistics of natural images and the response properties of cortical cells," J. Opt. Soc. Amer., vol. 4, no. 12, pp. 2379-2394, Dec. 1987.

[23] T. Pouli, D. Cunningham, and E. Reinhard, "Image statistics and their applications in computer graphics," in Proc. STAR, 2010, pp. 59-88.

[24] A. Gagalowitz and S. D. Ma, "Model driven synthesis of natural textures for 3-D scenes," Comput. Graph., vol. 10, no. 2, pp. 161-170, 1986.

[25] G. R. Cross and A. K. Jain, "Markov random field texture models," IEEE Trans. Pattern Anal. Mach. Intell., vol. PAMI-5, no. 1, pp. 25-39, Jan. 1983.

[26] J. A. Richards and X. Jia, "A Dempster-Shafer relaxation approach to context classification," IEEE Trans. Geosci. Remote Sens., vol. 45, no. 5, pp. 1422-1431, May 2007.

[27] G. J. McLachlan, "Mahalanobis distance," Resonance, vol. 4, no. 6, pp. 20-26, 1999.

[28] M. Marghany and M. Hashim, "Comparison between Mahalanobis classification and neural network for oil spill detection using RADARSAT-1 SAR data," Int. J. Phys. Sci., vol. 6, no. 3, pp. 566-576, Feb. 2011.

[29] K. Perumal and R. Bhaskaran, "Supervised classification performance of multispectral images," J. Comput., vol. 2, no. 2, pp. 124-129, Feb. 2010.

[30] B. Rodríguez-Cuenca, Clasificación de imagines con aplicación del contexto, Proyecto Fin de Carrera, Universidad de Alcalá, Spain, 2010.

[31] S. Zucker and J. Mohammed, "Analysis of probabilistic relaxation labeling processes," in Proc. IEEE Conf. Pattern Recog. Image Process., Chicago, IL, 1978, pp. 307-312.

[32] D. A. Landgrebe, "The development of a spectral-spatial classifier for Earth observational data," Pattern Recog., vol. 12, no. 3, pp. 165-175, 1980.

[33] J. A. Richards, D. A. Landgrebe, and P. H. Swain, "A means for utilizing ancillary information in multispectral classification," Remote Sens. Environ., vol. 12, no. 6, pp. 463-477, Dec. 1982.

[34] A. Gagalowitz, "A new method for texture field synthesis: Some applications to study of human vision," IEEE Trans. Pattern Anal. Mach. Intell., vol. PAMI-3, no. 5, pp. 520-533, Sep. 1981.

[35] A. Gagalowicz, "Texture modeling applications," Vis. Comput., vol. 3, no. 4, pp. 186-200, 1987

[36] A. Hyvärinen, J. Karhunen, and E. Oja, Independent Component Analysis. New York: Wiley, 2001.

[37] C. I. Chang, S. S. Chiang, J. A. Smith, and I. W. Ginsberg, "Linear spectral random mixture analysis for hyperspectral imagery," IEEE Trans. Geosci. Remote Sens., vol. 40, no. 2, pp. 375-392, Feb. 2002.
[38] X. Zhang and C. H. Chen, "New independent component analysis method using high order statistics with application to remote sensing images," Opt. Eng., vol. 41, no. 7, pp. 1717-1728, Jul. 2002.

[39] H. Shahbazi, P. Kabiri, and M. Soryani, "Content based multispectral image retrieval using independent component analysis," in Proc. Congr. Image Signal Process., 2008, pp. 485-489.

[40] M. S. Karoui, Y. Deville, S. Hosseini, A. Ouamri, and D. Ducrot, "Improvement of remote sensing multispectral image classification by using independent component analysis," in Proc. 1st WHISPERS, 2009, pp. 1-4.

[41] Q. Du, I. Kopriva, and H. Szu, "Independent-component analysis for hyperspectral remote sensing imagery classification," Opt. Eng., vol. 45, no. 1 , pp. 170081-1-170081-3, 2006

[42] J. A. Malpica, "Hue adjustment to IHS pan-sharpened IKONOS imagery for vegetation enhancement," IEEE Int. J. Geosci. Remote Sens. Lett., vol. 4, no. 1, pp. 27-31, Jan. 2007.

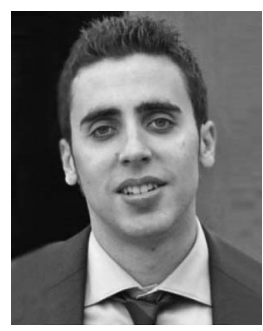

Borja Rodríguez-Cuenca (S'12) was born in Gijón, Spain, in 1987. He received the B.S. degree in topography from the University of Oviedo, Oviedo, Spain, in 2008 and the M.S. degree in geodesy and cartography from the University of Alcalá, Alcalá de Henares, Spain, in 2010, where he is currently working toward the Ph.D. degree.

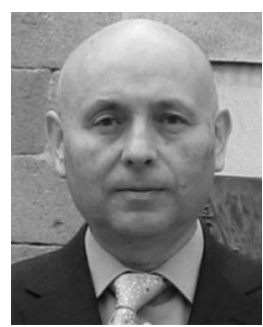

Jose A. Malpica received the M.S. and Ph.D. degrees in mathematics from Complutense University of Madrid, Madrid, Spain, in 1981 and 1992 respectively.

From 1998 to 2004, he was the Dean of the School of Cartography and Geodesy, University of Alcalá, Alcalá de Henares, Spain. He is currently an Associate Professor with the Department of Mathematics, University of Alcalá. Recently, he has also been actively involved in mathematics modeling for remote sensing phenomena, working in the Geodesy and Cartography Research Team of Alcalá. His current research interests are in multispectral and hyperspectral image analysis, neural network, and genetic algorithms with focus on feature extraction applications.

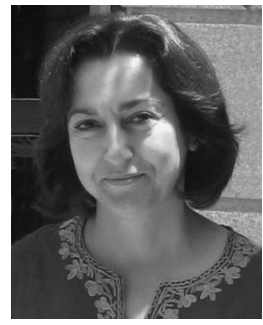

Maria C. Alonso received the B.S. and M.S. degrees in management science and statistics from Complutense University of Madrid, Madrid, Spain, in 1983 and 1985, respectively, and the Ph.D. degree in mathematics from the University of Alcalá, Alcala de Henares, Madrid, Spain, in 2003.

She is currently an Associate Professor with the Department of Mathematics, University of Alcalá. She has performed statistics consulting for industry, and she has worked on many projects involving pattern recognition for satellite image analysis. For the last 15 years, she has been working in the Geodesy and Cartography Research Team of Alcalá. 